\title{
1 On the role of photoreceptor identity in controlling accurate wiring of the
}

\section{Drosophila visual circuit}

3

4 Weiyue $\mathrm{Ji}^{1}$, Lani F. Wu ${ }^{2 *}$, Steven J. Altschuler ${ }^{2,3^{*}}$

5

6 Biophysics Graduate Group, University of California, San Francisco, San Francisco, CA 94158,

7 USA.

$8{ }^{2}$ Department of Pharmaceutical Chemistry, University of California, San Francisco, San Francisco,

9 CA 94158, USA

$10{ }^{*}$ Correspondence to: lani.wu@ucsf.edu, steven.altschuler@ucsf.edu 


\section{Abstract}

13 During development, neurons extend in search of synaptic partners. Precise control of axon

14 extension velocity can therefore be crucial to ensuring proper circuit formation. How velocity is

15 regulated - particularly by the extending axons themselves - remains poorly understood. Here, we

16 investigate this question in the Drosophila visual system, where photoreceptors make precise

17 connections with a specific set of synaptic partners that together create a circuit underpinning

18 neural superposition (NSP). We used a combination of genetic perturbations and quantitative

19 image analysis to investigate the influence of cell identity on growth cone velocity and subsequent

20 spatial-temporal coincidence of presynaptic and postsynaptic neurons. Our study provides a case

21 study of how cell autonomous properties of presynaptic axons play a pivotal role in controlling the

22 dynamics of growing axons and determining the formation of a precise neuronal circuit. 


\section{Introduction}

One of the most fascinating questions in neuroscience is how complex neural circuits form.

27 During development, neurons choose synaptic partners within brief time intervals. How do they

28 arrive at the right place and at the right time? One way to ensure proper circuit formation is through

29 precise control of extending growth cone velocities. Previous studies have shown that regulation

30 of velocity can be achieved through external signals, such as neurotransmitters and morphogens

31 (Castellani, 2013; Petros et al., 2008). However, the degree to which neurons autonomously

32 regulate their velocity is less well understood.

The Drosophila visual system offers a remarkable opportunity to investigate cell-

34 autonomous control of growth cone extension. Each eye comprises 800 unit eyes, or ommatidia,

35 and each ommatidium contains 8 photoreceptors ("R cells", labeled R1 through R8). Axons of the

36 photoreceptors extend from the eye to the optic lobe of Drosophila in groups of 800 "bundles"

37 with canonical intra-bundle orientations. Six of the eight R cells (R1-R6) stop extending at the

38 lamina plexus and wire to their postsynaptic targets, the lamina monopolar cells ("L cells"). At

39 each bundle, the R1-R6 cells diverge towards six different L-cell targets; and, at each L-cell target,

40 R1-R6 cells converge, each originating from a different bundle (Figure S1A). This final complex,

41 yet highly stereotyped neuronal circuit topology is referred to as neural superposition (NSP) (Agi

42 et al., 2014; Ting \& Lee, 2007).

43 An intriguing discovery is that R1-R6 each exhibit relatively consistent and cell subtype-

44 specific velocity (i.e. direction and speed) during growth cone extension (Langen et al., 2015).

45 This suggests that the velocity of growth cones plays a pivotal role in their synaptic partner

46 selection. Previous studies suggested that both ommatidia orientation of the originating bundle

47 (Clandinin \& Zipursky, 2000) and interactions among growth cones within the same bundle (Chen 
\& Clandinin, 2008; Schwabe et al., 2013) contribute to the direction of R cell projections. However,

49 no studies have investigated the role of a photoreceptor's identity in controlling its own growth

50 cone extension.

51 In this study, we used a combination of genetic perturbations and quantitative image

52 analysis to study the role of cell identity in controlling accurate wiring of the Drosophila visual

53 circuit. First, we utilized the local regularity of the circuit to develop a data-driven standardized

54 coordinate system around each bundle, which facilitated comparisons across samples. Then, we

55 quantified extension dynamics of wild-type photoreceptor growth cones across different

56 developmental stages. Next, we investigated how these dynamics change when cell fates of growth

57 cones are altered under genetic perturbations. Finally, we estimated the impact of nearest neighbor

58 interactions in determining extension velocity. Together, our quantitative study revealed that

59 photoreceptor identities determine their speed but not angle of extension, and that differences in

60 extension speed ultimately contribute to target specificity. Our study provides a case study of cell

61 autonomous mechanisms in neuronal circuit formation and highlights the importance of

62 disentangling the regulation of speed and direction - the two components of velocity - in

63 developmental processes.

\section{Results}

\section{Changes in $\mathbf{R 3}$ or $\mathbf{R} 4$ cell identity lead to changes in final targeting}

67 The role of photoreceptor identity during development has been extensively studied.

68 Photoreceptors develop in three sequential pairs during eye development: R2/R5, then R3/R4 and

69 last R1/R6 (Nériec \& Desplan, 2016; Treisman, 2013). The R3/R4 pair is particularly important, 
as the asymmetry between R3 and R4 determines the 90-degree rotation of R-cell clusters in the

71 developing eye disc and later, in the asymmetric trapezoidal arrangement of the adult ommatidia

72 (Treisman, 2013). Interestingly, the R3/R4 pair also stands out in the wiring diagram of NSP. The

73 target positions of R3 and R4 ("T3" and "T4", respectively) are asymmetrical, while the target

74 positions of the other two pairs (R1/R6, R2/R5) are symmetrical (Figure 1 - figure supplement 1,

75 A). Therefore, we focused our effort on understanding the role of R3/R4 identities-and their contribution to asymmetric targeting — during the NSP wiring process in the lamina.

To alter R3 and R4 cell identities, we used genetic perturbations in the planar-cell-polarity pathway. Specifically, over-expression of Frizzled $(F z)$ with sevenless $(\operatorname{sev})$ enhancer ( $\operatorname{sev}>F z)$ generates ommatidia with two R3s (Strutt et al., 1997; Zheng et al., 1995), while over-expression

80 of the intracellular domain of Notch $\left(N^{i c}\right)$ under the same enhancer $\left(\operatorname{sev}>N^{i c}\right)$ generates ommatidia

81 with two R4s (Cooper \& Bray, 1999; Fanto \& Mlodzik, 1999). To visualize the perturbed bundles,

82 we used a membrane-bound red fluorescent protein (RFP) under the same enhancer. Since the

83 lamina plexus is densely packed with photoreceptors, it is challenging to disambiguate individual

84 photoreceptor growth cones. Thus, we induced our perturbation sparsely. We utilized a FRT-

85 dependent GAL80 "flip-in" construct together with a heat-shock activated flippase to generate

86 sparse clones of perturbed bundles (Bohm et al., 2010; Chou \& Perrimon, 1996). Further, to

87 differentiate between R3s and R4s cell types at an early stage of development in both the

88 ommatidia and lamina, we utilized the R4-specific enhancer $m \delta 05$ fused with a membrane GFP

89 protein $(m \delta-G F P)($ Cooper \& Bray, 1999).

90 We visually inspected specimens of sparsely perturbed $\operatorname{sev}>F z$ and $\operatorname{sev}>N^{i c}$ flies after the

91 completion of NSP wiring ( $>36$ hours after puparium formation, or hrs APF; see Figure 1 - figure

92 supplement 1, B for timeline (Agi et al., 2014)). We found that changes in cell identity resulted in 
93 changes in final targeting (Figures 1, Figure 1 - figure supplement 2). For bundles with two R4

94 photoreceptors, both the normal and fate-transformed R4s target the canonical R4 target, T4. For

95 bundles with two R3 photoreceptors, the normal R3 targets the canonical R3 target, T3, while the

96 fate-transformed R3 targets T3', a new target position that is mirror-symmetric to T3, instead of

97 the original T3. (See Supplementary file 1A for phenotype penetrance.) This result raised the

98 hypothesis that cell identity interacts with bundle position to shape the targeting of R3 and R4

99 photoreceptors.

100

101 Wild-type R3s and R4s exhibit asymmetric speeds but symmetric directions of extension

102 Perturbing cell identity changed the final target choice. But, did it also change the behavior

103 of early extension? We next focused on quantitative characterization of growth cone extension 104 during NSP.

105 Characterizing patterns in growth cone extension requires comparing growth cone 106 morphology across multiple bundles and between animals. The wiring of the Drosophila lamina

107 exhibits a crystal-like two-dimensional pattern for the 800 bundles across the lamina plexus.

108 However, variations in image orientation and irregular local warping of the lamina plexus

109 confounds bundle-to-bundle and sample-to-sample comparison. We therefore developed an

110 approach that utilizes the local regularity of a growth cone's environment and quantifies its

111 behavior according to a standardized polar coordinate system (Figure 2; Methods). For each bundle,

112 we identified the starting positions of all R-cells (Heel grid, Figure 2A) and their putative targets

113 (Target grid, Figure 2B). We also identified a center point "C", which lies at the intersection of the

114 line connecting R3 and T3 and the line connecting R4 and T3'. We then extrapolated polar

115 coordinates by normalizing (Figure 2C): 1) length, so that $|\mathrm{C}-\mathrm{T} 4|=1$ (A.U.); and 2) angle, so that 
$116 \Varangle(\mathrm{T} 3, \mathrm{C}, \mathrm{T} 4)=\Varangle\left(\mathrm{T} 4, \mathrm{C}, \mathrm{T}^{\prime}{ }^{\prime}\right)=1$ (A.U.) and that $\mathrm{T} 3$ and $\mathrm{T} 3$ ' were placed at angles +1 and -1 ,

117 respectively. (We note that in our samples, $|\mathrm{C}-\mathrm{T} 4|$ is $7.3 \pm 1.5 \mu \mathrm{m}$ and $\Varangle\left(\mathrm{T} 3, \mathrm{C}, \mathrm{T} 33^{\prime}\right)$ is $27.8 \pm 6.8^{\circ}$.)

118 Using these standardized coordinates, we examined behavior of wild-type flies taken from

119 early to late stage of neural superposition ( $22 \mathrm{hrs}$ to $36 \mathrm{hrs})$ to characterize the dynamics of R3 and

120 R4 growth cones during extension. First, we examined changes in the morphologies of R3 and R4

121 growth cones over time. By $22 \mathrm{hrs}$ APF, growth cones of both R3s and R4s have already polarized

122 (Figures 3A and Figure 3 - figure supplement 1), with numerous filopodia at the leading edge.

123 This morphology is maintained until $\sim 32 \mathrm{hrs}$ APF, after which the number of filopodia decreases

124 and the growth cones take on narrower, more linear morphologies (Figure 3A).

125 Next, we examined the speed and angle of growth cones and their relationship with their

126 targets. The growth cone of R4 is close to T4 as early as $22 \mathrm{hrs} \mathrm{APF,} \mathrm{with} \mathrm{some} \mathrm{of} \mathrm{the} \mathrm{filopodia} \mathrm{at}$

127 the leading edge of the growth cone already appearing to make contact, and fully reach T4 by 28

128 hrs APF. R3 growth cones are initially similar in length to R4 at 22 hrs APF, yet they continue to

129 extend past T4 starting from $24 \mathrm{hrs}$ APF and reach the more distant target T3, by 28 hrs APF

130 (Figure 3B). Thus, R3s have a markedly higher speed of extension than R4s — a roughly 8-fold

131 difference (0.16 a.u./hour vs. 0.02 a.u./hour). While the speed of R3 and R4 are asymmetric, their

132 extension angles are, surprisingly, symmetrical at early stages of NSP wiring (Figure 3C). We

133 observed dramatic changes in the extension angle of R4s at $32 \mathrm{hrs}$ APF and R3s at $36 \mathrm{hrs}$ APF,

134 correlating with morphological changes that indicate the transition from axon pathfinding to

135 synapse formation. 


\section{Extension speed is responsible for asymmetrical targeting}

Based on these observations in wild-type, we hypothesized that extension speed is responsible for the asymmetrical targeting of R3/R4 pairs. To test this hypothesis, we examined

140 the behavior of sparsely perturbed mutant $\left(\operatorname{sev}>F z\right.$ and $\left.\operatorname{sev}>N^{i c}\right)$ flies that have both wild-type-like

141 and mutant bundles at 24 or $28 \mathrm{hrs}$ APF, corresponding to early or late stages of their extension

142 (Figures 4 and Figure 4 - figure supplement 1).

We compared the behaviors of fate-transformed to wild-type bundles. The fate-transformed

144 bundles contain either two R3s or two R4s (one of which is fate transformed and the other wild145 type). We observed that the extension speed and angle of the wild-type cells in the fate-transformed

146 bundles is similar to the $\mathrm{R}$ cells of the same identity in the wild-type-like bundles. More

147 importantly, we discovered that within the fate-transformed bundles, the two R cells extend at 148 similar speeds and symmetric angles (Figure 4). Thus, we conclude that cell identity is 149 instrumental in determining extension speed of photoreceptor growth cones, which ultimately lead 150 to the asymmetrical targeting of R3 and R4s.

Taken together, the initial directions of extension for R-cells at the R3 and R4 positions are

152 mirror symmetric and independent of cell identity (i.e., whether wild-type or fate-transformed).

153 What could determine the initial extension directions? Simulations based on the geometric 154 configurations of R-cell bundles and their targeting positions (Langen et al., 2015) suggested the 155 possibility of a simple repulsion rule. That is, equal repulsion from immediate neighbor(s) within 156 the same bundle determines the extension direction of an $\mathrm{R}$ cell. While this appeared reasonable 157 for most of the R cells, R3/4 have asymmetrical targets and it was unclear how this rule could 158 apply to their initial extension directions. In light of the discovery that R3 and R4 initially extend 159 symmetrically, we re-examined the possibility of a simple repulsion rule (Figure 4 - figure 
160 supplement 2, A). Analysis showed that this would lead to predicted extension angles that are $\sim 30^{\circ}$

161 further from the bundle midline than those that are experimentally measured (Figure 4 - figure

162 supplement 2, B). Instead, regression analysis suggested that R2 or R5 contribute $\sim 2 \mathrm{x}$ the repulsion

163 of the other immediate neighbor R3 or R4 (e.g. for the case of R3, R2 has twice the repulsion of

164 R4) (Figure 4 - figure supplement 2, B). Thus, our quantitative study of growth cone extension

165 suggests the hypothesis that a weighted repulsion model among neighbors within a bundle 166 determines extension directions during patterning of the NSP circuit.

\section{Discussion}

The velocity, defined by both direction and speed of the growth cone, is an important

170 property of extending neurons during circuit formation. How is velocity controlled during neuronal

171 development, and what are the consequences of changes in velocity in vivo for circuit formation?

172 Here, we investigate this question in the context of Drosophila NSP circuit, in which $\sim 4800$

173 neurons (=800 bundles x 6 R-cell types) swap relative positions and identify their targets with

174 astonishing accuracy (Horridge \& Meinertzhagen, 1970). To compare the dynamics across

175 populations of neurons, we developed a standardized coordinate system for describing growth cone

176 position. This coordinate system was essential in overcoming bundle-to-bundle and fly-to-fly

177 heterogeneity, and similar approaches could be adapted to quantify and compare the dynamics of

178 neurons in other developmental systems with other wiring geometries. We found that cell identities

179 for R3 and R4 neurons determine their speed but not direction of extension. As a consequence,

180 while R3 and R4 start with symmetric extension directions, their differences in speed lead to

181 extension length differences by the final time of wiring and subsequently to asymmetric target

182 choices. These observations highlight a crucial role for cell-autonomous mechanisms in 
183 controlling the dynamics of neuronal extension and, ultimately, the spatial-temporal coincidence

184 of presynaptic and postsynaptic neurons.

185 One simple self-organizing mechanism to determine extension direction of photoreceptor

186 growth cones is through repulsion of neighboring growth cones within the same bundle. We

187 discovered that a simple repulsion model — where each R cell contributes equally — is not sufficient

188 to explain the measured extension direction; rather, a model is supported in which R2/5 contributes

189 much more strongly than R3/4. Such differential repulsion could arise through different strengths

190 of expressed repulsive cues among neighboring cells. Interestingly, previous studies did show that

191 Flamingo, a seven-pass transmembrane cadherin capable of inducing repulsion in both axons and

192 dendrites (Matsubara et al., 2011; Senti et al., 2003), is differentially expressed amongst the

193 different R-cell types, with R2/R5 having $\sim 3 x$ the expression levels of R3/R4 (Schwabe et al.,

194 2013). Our study provides a quantitative framework to investigate molecular mechanisms

195 underlying this developmental decision process.

Our study also provided a functional readout and framework for future investigations into

197 mechanisms of how cell identity controls growth cone dynamics. Regulation of cytoskeletal

198 dynamics is a clear possibility. Studies in cultured mammalian neurons have identified key

199 components of the cytoskeleton that regulate axon outgrowth and how these components can be

200 regulated by signaling molecules (Dent et al., 2011; Geraldo \& Gordon-Weeks, 2009). For

201 example, the cell-surface receptor Notch has been shown to regulate the speed of neurite outgrowth

202 (Berezovska et al., 1999; Franklin et al., 1999; Šestan et al., 1999) via controlling the stability of

203 microtubules (G. Ferrari-Toninelli et al., 2008) and the expression levels of signaling proteins

204 (Giulia Ferrari-Toninelli et al., 2009). In the Drosophila NSP circuit, there is evidence that

205 cytoskeletal regulators, such as Genghis khan and $\mathrm{Cdc} 42$, plays a crucial role in precise targeting 
of photoreceptors (Gontang et al., 2011). Additionally, while we focused on extension velocity,

207 our findings do not exclude other factors that may also contribute to growth cone wiring that are

208 also changed when cell identity is transformed. Understanding how cell identities translate to

209 differences in molecular profiles and finally to changes in the cytoskeletal networks that control

210 growth cone dynamics, will provide valuable insight into how growth cone velocity can be

211 controlled during neuronal circuit development in vivo.

212 Changes in neurite speed have been observed in multiple models of neuronal circuit

213 formation. For example, axons that require midline crossing often exhibit large changes in growth

214 cone kinetics adjacent to the midline. Such pausing behavior is observed in crossing of mice retinal

215 axons (Godement et al., 1994; Mason \& Wang, 1997), zebrafish retinal ganglion cells (Hutson \&

216 Chien, 2002), and zebrafish postoptic commissures (Bak \& Fraser, 2003). Growth cones of

217 Zebrafish Mauthner cells also pause briefly when encountering successive motor neurons while

218 extending along the midline (Jontes et al., 2000). There are also examples of changes in neurite

219 velocity that have functional consequences both in vitro and in vivo. In studies of cultured snail

220 Helisoma neurons, it has been shown that changes in ganglion neuronal speed correlate with their

221 ability to form electrical synapses among themselves (P. Haydon et al., 1984; P. G. Haydon et al.,

222 1987). Furthermore, there is evidence in both vertebrate and invertebrate sensory circuits that

223 direction and speed of axon-dendritic interactions can contribute to connection specificity

224 (Balaskas et al., 2019; Kiral et al., 2020). Our study in the Drosophila neural superposition

225 demonstrates how cell-autonomous control of velocity is instrumental in the precise wiring of a

226 complex sensory circuit. Taken together, these studies highlight the importance of velocity and

227 vector-based strategies in developmental neuroscience. 


\section{Materials and methods}

\section{$230 \quad$ Fly stocks and handling}

231 Fly stocks were constructed and maintained at $25{ }^{\circ} \mathrm{C}$ using standard protocols.

232 The following fly lines were used in this study: tubP $>$ stop $>$ GAL80 (II), tubP $>$ stop $>$ GAL80 (III)

233 and hs-FLP122 (X) (gifts from T. Clandinin, Stanford University), m805-CD4::GFP (III) (gift

234 from P.R. Hiesinger, Free University Berlin), Uas-N ${ }^{i c}$ (II) (gift from B.A. Hassan, ICM Institute

235 for Brain and Spinal Cord); UAS-CD4::tdTomato (II) (gift from L.Y. Jan and Y.N. Jan, UCSF),

236 UAS-fz1-1 (Bloomington Drosophila Stock Center \# 41791) and sevEP-GAL4.B (II)

237 (Bloomington Drosophila Stock Center \# 5793).

238 For experiments with pupae samples, pupae with the correct genotype were collected at 0 hrs after

239 puparium formation (APF) and aged at $28^{\circ} \mathrm{C}$ to increase the penetration of genetic perturbations

240 (Das et al., 2002). For experiments with adult samples, flies 3-5 days after eclosion were collected.

241 Both male and female were used for all experiments.

243 Heat shock clone induction

244 Larvae with the correct genotype were heat shocked for $8-15$ mins at $37{ }^{\circ} \mathrm{C}$ at 2 to 4 days after 245 egg laying (AEL).

247 Immunohistochemistry and Imaging of Pupae Brains

$248 \quad$ Dissection and Staining

249 For preparation of pupal brain samples, pupal brains were dissected at the appropriate 250 developmental stages in PBS (Phosphate Buffered Saline) and fixed with 3.7\% formaldehyde-PBS

251 for 30 mins. Fixed brains were washed three times in PBT (PBS with $0.4 \%$ Triton X-100) at room 
252 temperature and then blocked with PBT-BSA (3\% Bovine Serum Albumin in PBT) for 1 hour.

253 Two rounds of antibody staining were then performed. In each round, brains were incubated with

254 cocktails of primary antibodies at $4^{\circ} \mathrm{C}$ overnight, rinsed in PBT, then incubated with cocktails of

255 secondary antibodies at $4^{\circ} \mathrm{C}$ overnight or for $2 \mathrm{hrs}$ at room temperature, then rinsed again in PBT.

256 For preparation of adult eye samples, eyes were dissected in PBS and fixed with 3.7\%

257 formaldehyde-PBS for 30 mins. Fixed samples were washed three times in PBT (PBS with $0.4 \%$

258 Triton X-100) and kept at room temperature in PBT for up to 12 hrs to reduce the pigmentation of

259 eyes. Samples were then incubated with 1:50 Alexa Fluor 633 phalloidin (A22284, ThermoFisher

260 Scientific) for $2 \mathrm{hrs}$ at room temperature and rinsed in PBT.

$261 \quad$ Antibodies

262 Primary antibodies used for the first round are: mouse anti-Fasciclin II (DSHB, 1D4), 1:20;

263 Chicken anti-GFP (abcam, ab13970), 1:400; Rabbit anti-RFP (Rockland), 1:400. Conjugated

264 secondary antibodies used for the first round are: Goat anti-mouse Alexa-405 (A31553, Thermo

265 Fisher Scientific), 1:250; Goat anti-chicken Alexa-488 (A32931, Thermo Fisher Scientific), 3:500;

266 Donkey anti-rabbit Alexa-568 (A10042, Thermo Fisher Scientific), 1:500. Primary antibody used

267 for the second round is: mouse anti-Chaoptin (DSHB, 24B10), 1:20. Conjugated secondary

268 antibody used for the second round is: Donkey anti-mouse Alexa-647 (A31571, Thermo Fisher

269 Scientific), 1:100.

$270 \quad$ Mounting and Imaging

271 Samples were mounted in VECTASHIELD Antifade Mounting Medium. Images were obtained

272 on a Nikon A1R-Si inverted confocal microscope with 4 line laser unit (405/488/561/640) and

273 with a $60 \mathrm{X}$ oil objective. $\mathrm{Z}$ stacks were acquired with a step size of $0.125 \mu \mathrm{m}$ between optical

274 sections. 


\section{Image Processing for Visual Inspection and Figure Generation}

276 Images were transformed from ND2 format to TIF format and background subtracted in batch

277 using custom ImageJ macro script on a HPC cluster (code available on GitHub).

278 To calculate the penetration of $\operatorname{sev}>F z$ and $s e v>N^{i c}$ perturbation (Table S1), images of $\operatorname{sev}>F z$

279 lamina samples at $38 \mathrm{hrs}$ APF and sev $>\mathrm{N}^{\mathrm{ic}}$ lamina samples at $45 \mathrm{hrs}$ APF were visually inspected

280 using Fiji (http://fiji.sc/) and targets of bundles with two R3s (bundles with two RFP-positive R

281 cell growth cones and no GFP-positive R cell growth cones) or two R4s (bundles with two GFP-

282 positive $\mathrm{R}$ cell growth cones) are counted. Only bundles with R cell growth cones that can be easily

283 traced from origin to target are included in the counting. Fz and Nic perturbation were scored at

284 different developmental time points due to the decay of quality of GFP signal in $s e v>F z$ samples

285 over time.

286 To generate representative images of bundle targeting phenotypes (Figure 1 and Figure 1 - figure

287 supplement 2), we annotated cropped confocal images of $s e v>F z$ samples at 38 hrs APF and $288 s e v>N^{i c}$ samples at $45 \mathrm{hrs}$ APF in Amira 2020.1 (FEI Visualization Sciences Group). For each

289 cropped image stack, growth cones from one bundle were manually segmented in both GFP and

290 RFP channels. Segmented growth cones were then rendered with the appropriate color (red for

291 RFP and green for GFP) in volume, and the unsegmented FasII channel was overlaid as an ortho-

292 slice in gray-scale. TIF files of the results were then exported from Amira and further annotated

293 using Adobe Illustrator. To show bundles in consistent orientations, some images were rotated

294 and/or flipped. Images were also cropped to highlight the representative bundle.

295 To generate representative images of bundles from 22 to $36 \mathrm{hrs}$ APF (Figure 2, Figure 3A, Figure

2963 - figure supplement 1 and Figure 4 - figure supplement 1), images of lamina samples were

297 inspected and adjusted using Fiji (http://fiji.sc/). Brightness and contrast of individual channels 
were adjusted separately, and only one z-stack was selected for visualization purpose. Images are

299 also cropped to highlight the representative bundle. Tiff files of individual channels were exported

300 from Fiji and further annotated using Adobe Illustrator. To show bundles in consistent orientations,

301 some images were rotated and/or flipped. Images were also cropped to highlight the representative

302 bundle.

303

304 Image Quantification Using Standardized Coordinates

305 Pre-preprocessing

306 Images of laminas were transformed from ND2 format to TIF format and background subtracted

307 in batch using custom ImageJ macro script on a HPC cluster (code available on GitHub).

308 Annotation

309 Images were then visually inspected, cropped and annotated using Fiji (http://fiji.sc/). Due to the

310 variation in mounting and sparseness of labeling, only images of the lamina plexus with large

311 intact regions were further analyzed. Images were cropped (in all directions) to keep the part of

312 the lamina plexus that had sparse-enough labeling of growth cones. These cropped images were

313 then used to manually annotate the position of growth cone heels (starting positions) and targets

314 using the Multi-point tool. Heels of growth cones were positioned based on the 24B10 channel

315 while targets were positioned based on the FasII channel. Heels are annotated with points while

316 targets are annotated with ellipses. Multiple z-slices were used to annotate target ellipses to better

317 represent the boundaries of FasII staining. X, Y, positions of heels and major, minor axis lengths

318 and the major axis angle of the target ellipse were exported to a csv file for later quantification.

319 Mapping of each bundle number to its corresponding target numbers was also noted in another csv

320 file. Bundles with rotational defects were not included in the annotation. 


\section{Quantification}

322 Standardized coordinate system. We used custom Python scripts (code available on GitHub) to

323 resample GFP and RFP images of each bundle to obtain representative density maps according to

324 a standardized coordinate. Our standardized coordinate system (see Figure 2) resembles the polar

325 coordinate system. The center of the coordinate system $(C=(0,0))$ is defined as the intersection of

326 the lines connecting R3 and T3 and R4 and T3'. The polar coordinate is normalized so that: 1) the

327 radius $|\mathrm{C}-\mathrm{T} 4|=1$ (A.U.) and 2) the targets $\mathrm{T} 3$ and $\mathrm{T} 3$ ' are placed at angles +1 and -1 (A.U.),

328 respectively. The centers of the target ellipses were used as reference points for the standardized

329 coordinate system.

330 Density map of image slice. We converted image data to a density map in our standardized

331 coordinates in two steps. First, we created a coordinate grid (the radius ranged from 0 to 3.8 with

3320.05 intervals; the angles ranged from -3 to 3 with 0.05 intervals). Second, we used the N-

333 dimensional piecewise linear interpolation function within the Python numpy package (v1.16.4)

334 to create a map from Cartesian to polar coordinates; this allowed us to convert GFP and RFP

335 images into density maps in the new coordinate system.

336 Density map of bundle. Density maps for each bundle were computed using the mean density map

337 across z-stacks containing 41 slices, which were centered around the z-slice showing the longest

338 growth cone (typically R3 and/or R4). We manually annotated the length and angle of R3 or R4

339 growth cones of a given bundle according to the RFP or GFP density map, respectively. Length

340 and angle of growth cones were annotated based on the longest filopodia in the front of the growth

341 cone. Growth cones located in regions where GFP or RFP signals was to dense to distinguish the

342 front were excluded from the annotation. If growth cones exhibit split morphology (i.e., two or

343 more major long filopodia in the front), angles are calculated by the mean of these filopodia. 
344 Growth cones were labeled as R3 or R4 based on the absence or presence (respectively) of GFP

345 signals.

347 Simulation of Repulsion Model

348 Heel and target positions were mapped to the standardized coordinate system. Simulated extension 349 angles $\left(\overrightarrow{v_{p}}\right)$ were calculated based on weighted vector sum of two vectors $\left(\overrightarrow{v_{1}}, \overrightarrow{v_{2}}\right)$. For extension 350 angles of $\mathrm{R} 3$, the repulsion vectors: $\overrightarrow{v_{1}}$ was taken to be the unit vector from the R2 to R3 heels; 351 and $\overrightarrow{v_{2}}$ was taken to be the unit vector from the R4 to R3 heels. For extension angles of R4, the 352 repulsion vectors: $\overrightarrow{v_{1}}$ was taken to be the unit vector from the R5 to R4 heels; and $\overrightarrow{v_{2}}$ was taken to 353 be the unit vector from the R3 to R4 heels. The weight of each vector represented the strength of 354 its repulsive force. For simple repulsion from neighboring heels, $\overrightarrow{v_{p}}=\left(\overrightarrow{v_{1}}+\overrightarrow{v_{2}}\right)$. To estimate 355 unequal influence of neighboring growth cone heels, linear regression was performed on $\overrightarrow{v_{p}}=\alpha$ $356 \overrightarrow{v_{1}}+\beta \overrightarrow{v_{2}}$ using pooled data from wild-type measurements between 22 to $26 \mathrm{hrs}$ APF. Only data

357 from bundles that were relatively symmetric in shape $\left(\frac{\left|\Varangle(\mathrm{T} 3, \mathrm{C}, \mathrm{T} 4)-\measuredangle\left(\mathrm{T} 33^{\prime}, \mathrm{C}, \mathrm{T} 4\right)\right|}{\max \left\{\measuredangle(\mathrm{T} 3, \mathrm{C}, \mathrm{T} 4), 4\left(\mathrm{~T} 3^{\prime}, \mathrm{C}, \mathrm{T} 4\right)\right\}} \leq 0.5\right)$ were 358 included in the regression analysis. R3 and R4 angles were fitted independently. Regression 359 analysis was implemented in Python using the scikit-learn package (v0.23.2).

\section{Statistical Analysis}

362 Sample sizes for each experiment are provided in the figure legends. Statistics were computed in 363 Python using the scipy (v1.2.1) and scikit-posthocs (v0.6.4) packages. A two-sided Mann-Whitney $364 \mathrm{U}$ test was applied when there were only two groups of data being compared. When there were 365 more than two groups, we applied a Kruskal-Wallis H test followed by a post-hoc two-sided 366 Mann-Whitney test with $\mathrm{p}$ values adjusted by Bonferroni method. The error bars displayed in all 
367 figures represent standard deviation of the mean. Data collection and analysis were not conducted

368 blind to the conditions of the experiments.

369

370 Acknowledgements

371 We gratefully acknowledge helpful discussions and feedback from Thomas R. Clandinin, Graeme

372 Davis, Marion Langen, and Orion Weiner during the project. We also wish to thank the Clandinin,

373 Hassan, Hiesinger, and Jan labs for fly lines. Finally, we thank Peter Robin Hiesinger, Egemen

374 Agi, and members of the Altschuler-Wu lab and Hiesinger lab for critical comments on the

375 manuscript.

376

\section{Author Contributions}

378 Conceptualization and Methodology, W.J., S.J.A. and L.F.W.; Investigation, Formal Analysis, and

379 Visualization, W.J.; Writing - Original Draft, W.J.; Writing - Review \& Editing, W.J., S.J.A. and

380 L.F.W.; Supervision, S.J.A. and L.F.W.

381

382 Competing Interests Statement

383 The authors declare no competing interests. 


\section{References}

386 Agi, E., Langen, M., Altschuler, S. J., Wu, L. F., Zimmermann, T., \& Hiesinger, P. R. (2014).

387 The Evolution and Development of Neural Superposition. Journal of Neurogenetics, 28(3-4),

388 216-232. https://doi.org/10.3109/01677063.2014.922557

Bak, M., \& Fraser, S. E. (2003). Axon fasciculation and differences in midline kinetics between pioneer and follower axons within commissural fascicles. Development, 130(20), 4999-5008. https://doi.org/10.1242/dev.00713

Balaskas, N., Abbott, L. F., Jessell, T. M., \& Ng, D. (2019). Positional Strategies for Connection 1156.e4. https://doi.org/10.1016/j.neuron.2019.04.008

Berezovska, O., McLean, P., Knowles, R., Frosh, M., Lu, F. M., Lux, S. E., \& Hyman, B. T. (1999). Notch1 inhibits neurite outgrowth in postmitotic primary neurons. Neuroscience, 93(2), 433-

Bohm, R. A., Welch, W. P., Goodnight, L. K., Cox, L. W., Henry, L. G., Gunter, T. C., Bao, H., 439. https://doi.org/10.1016/s0306-4522(99)00157-8 \& Zhang, B. (2010). A genetic mosaic approach for neural circuit mapping in Drosophila. Proceedings of the National Academy of Sciences, 107(37), 16378-16383. https://doi.org/10.1073/pnas.1004669107 
404 Chen, P.-L., \& Clandinin, T. R. (2008). The Cadherin Flamingo Mediates Level-Dependent 405 Interactions that Guide Photoreceptor Target Choice in Drosophila. Neuron, 58(1), 26-33. 406 https://doi.org/10.1016/j.neuron.2008.01.007

407 Chou, T. B., \& Perrimon, N. (1996). The autosomal FLP-DFS technique for generating germline 408 mosaics in Drosophila melanogaster. Genetics, 144(4), 1673-1679.

409 Clandinin, T. R., \& Zipursky, S. L. (2000). Afferent Growth Cone Interactions Control Synaptic 410 Specificity in the Drosophila Visual System. Neuron, 28(2), 427-436. 411 https://doi.org/10.1016/s0896-6273(00)00122-7

412 Cooper, M. T. D., \& Bray, S. J. (1999). Frizzled regulation of Notch signalling polarizes cell fate 413 in the Drosophila eye. Nature, 397(6719), 526-530. https://doi.org/10.1038/17395

414 Das, G., Reynolds-Kenneally, J., \& Mlodzik, M. (2002). The Atypical Cadherin Flamingo Links 415 Frizzled and Notch Signaling in Planar Polarity Establishment in the Drosophila Eye. 416 Developmental Cell, 2(5), 655-666. https://doi.org/10.1016/s1534-5807(02)00147-8

417 Dent, E. W., Gupton, S. L., \& Gertler, F. B. (2011). The Growth Cone Cytoskeleton in Axon 418 Outgrowth and Guidance. Cold Spring Harbor Perspectives in Biology, 3(3), a001800. 419 https://doi.org/10.1101/cshperspect.a001800

420 Fanto, M., \& Mlodzik, M. (1999). Asymmetric Notch activation specifies photoreceptors R3 and 421 R4 and planar polarity in the Drosophila eye. Nature, 397(6719), 523-526. 422 https://doi.org/10.1038/17389 
423 Ferrari-Toninelli, G., Bonini, S. A., Bettinsoli, P., Uberti, D., \& Memo, M. (2008). Microtubule stabilizing effect of notch activation in primary cortical neurons. Neuroscience, 154(3), 946952. https://doi.org/10.1016/j.neuroscience.2008.04.025

Ferrari-Toninelli, Giulia, Bonini, S. A., Uberti, D., Napolitano, F., Stante, M., Santoro, F., Minopoli, G., Zambrano, N., Russo, T., \& Memo, M. (2009). Notch activation induces neurite remodeling and functional modifications in SH-SY5Y neuronal cells. Developmental A., \& Nye, J. S. (1999). Autonomous and non-autonomous regulation of mammalian neurite development by Notch1 and Delta1. Current Biology, 9(24), 1448-1457. Neurobiology, 69(6), 378-391. https://doi.org/10.1002/dneu.20710

Godement, P., Wang, L., \& Mason, C. (1994). Retinal axon divergence in the optic chiasm: https://doi.org/10.1016/s0960-9822(00)80114-1 1995 Mar;15(3):following table of contents]. The Journal of Neuroscience, 14(11), 7024-7039. https://doi.org/10.1523/jneurosci.14-11-07024.1994

Gontang, A. C., Hwa, J. J., Mast, J. D., Schwabe, T., \& Clandinin, T. R. (2011). The cytoskeletal regulator Genghis khan is required for columnar target specificity in the Drosophila visual system. Development, 138(22), 4899-4909. https://doi.org/10.1242/dev.069930 
443 Haydon, P. G., McCobb, D. P., \& Kater, S. B. (1987). The regulation of neurite outgrowth, growth 444 cone motility, and electrical synaptogenesis by serotonin. Journal of Neurobiology, 18(2), 197445 215. https://doi.org/10.1002/neu.480180206

446 Haydon, P., McCobb, D., \& Kater, S. (1984). Serotonin selectively inhibits growth cone motility 447 and synaptogenesis of specific identified neurons. Science, 226(4674), 561-564. $448 \quad$ https://doi.org/10.1126/science.6093252

Horridge, G. A., \& Meinertzhagen, I. A. (1970). The accuracy of the patterns of connexions of the 450 first- and second-order neurons of the visual system of Calliphora. Proceedings of the Royal Society of London. Series B. Biological Sciences, 175(1038), 69-82. Role of astray/robo2. Neuron, 33(2), 205-217. https://doi.org/10.1016/s0896-6273(01)00579https://doi.org/10.1098/rspb.1970.0012 embryos: early events in synaptogenesis imaged in vivo. Nature Neuroscience, 3(3), 231-237. synaptic partner choice during Drosophila brain wiring. Nature Communications, 11(1), 1325. https://doi.org/10.1038/s41467-020-14781-4 
463 Langen, M., Agi, E., Altschuler, D. J., Wu, L. F., Altschuler, S. J., \& Hiesinger, P. R. (2015). The

464 Developmental Rules of Neural Superposition in Drosophila. Cell, 162(1), 120-133.

465 https://doi.org/10.1016/j.cell.2015.05.055

466 Mason, C. A., \& Wang, L.-C. (1997). Growth Cone Form Is Behavior-Specific and, Consequently,

467 Position-Specific along the Retinal Axon Pathway. The Journal of Neuroscience, 17(3), 1086-

468 1100. https://doi.org/10.1523/jneurosci.17-03-01086.1997

469 Matsubara, D., Horiuchi, S., Shimono, K., Usui, T., \& Uemura, T. (2011). The seven-pass

470 transmembrane cadherin Flamingo controls dendritic self-avoidance via its binding to a LIM

471 domain protein, Espinas, in Drosophila sensory neurons. Genes \& Development, 25(18), 1982-

472 1996. https://doi.org/10.1101/gad.16531611

473 Nériec, N., \& Desplan, C. (2016). From the Eye to the Brain: Development of the Drosophila

474 Visual System. Current Topics in Developmental Biology, 116, 247-271.

475 https://doi.org/10.1016/bs.ctdb.2015.11.032

476 Petros, T. J., Rebsam, A., \& Mason, C. A. (2008). Retinal Axon Growth at the Optic Chiasm: To

477 Cross or Not to Cross. Annual Review of Neuroscience, 31(1), 295-315.

478 https://doi.org/10.1146/annurev.neuro.31.060407.125609

479 Schwabe, T., Neuert, H., \& Clandinin, T. R. (2013). A Network of Cadherin-Mediated Interactions

480 Polarizes Growth Cones to Determine Targeting Specificity. Cell, 154(2), 351-364.

$481 \quad$ https://doi.org/10.1016/j.cell.2013.06.011 
482 Senti, K.-A., Usui, T., Boucke, K., Greber, U., Uemura, T., \& Dickson, B. J. (2003). Flamingo

483 Regulates R8 Axon-Axon and Axon-Target Interactions in the Drosophila Visual System.

484 Current Biology, 13(10), 828-832. https://doi.org/10.1016/s0960-9822(03)00291-4

485 Šestan, N., Artavanis-Tsakonas, S., \& Rakic, P. (1999). Contact-Dependent Inhibition of Cortical

486 Neurite Growth Mediated by Notch Signaling. Science, 286(5440), 741-746.

$487 \quad$ https://doi.org/10.1126/science.286.5440.741

488 Strutt, D. I., Weber, U., \& Mlodzik, M. (1997). The role of RhoA in tissue polarity and Frizzled

489 signalling. Nature, 387(6630), 292-295. https://doi.org/10.1038/387292a0

490 Ting, C.-Y., \& Lee, C.-H. (2007). Visual circuit development in Drosophila. Current Opinion in

$491 \quad$ Neurobiology, 17(1), 65-72. https://doi.org/10.1016/j.conb.2006.12.004

492 Treisman, J. E. (2013). Retinal differentiation in Drosophila. Wiley Interdisciplinary Reviews:

493 Developmental Biology, 2(4), 545-557. https://doi.org/10.1002/wdev.100

494 Zheng, L., Zhang, J., \& Carthew, R. W. (1995). frizzled regulates mirror-symmetric pattern 495 formation in the Drosophila eye. Development (Cambridge, England), 121(9), 3045-3055. 

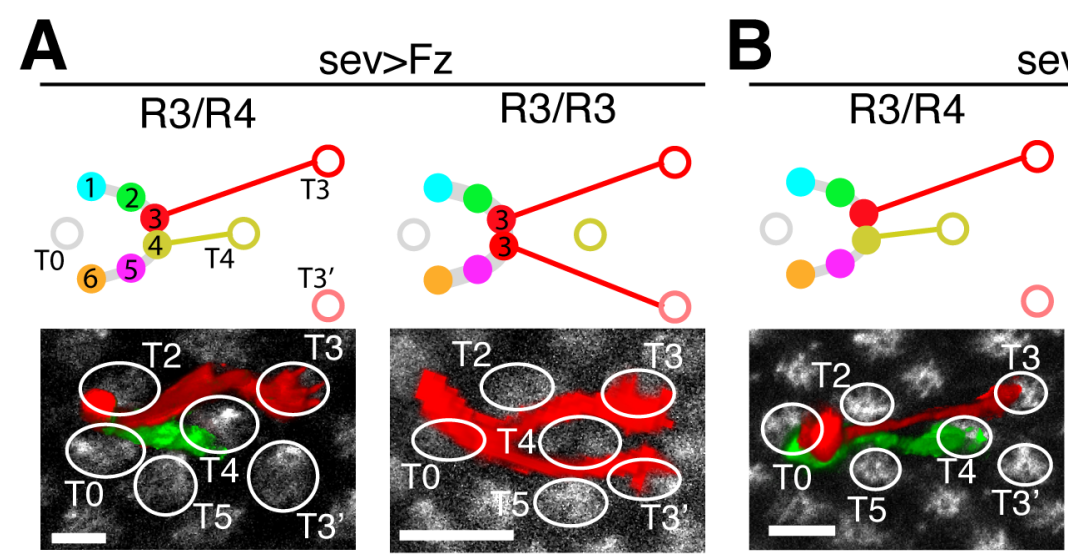

sev $>N^{\text {ic }}$

38 hrs APF

R3/R4, R4

45hrs APF

3 Figure 1. Changes in R3 or R4 cell identity lead to changes in final targeting.

4 (A-B) Representative schematics and images of bundles from (A) $\operatorname{sev}>F z$ (38 hrs APF) and (B)

$5 \operatorname{sev}>N^{i c}(45 \mathrm{hrs}$ APF) flies (see Figure 1 - figure supplement 2 for more examples). Top panels:

6 schematic of wild-type or altered wiring topology. Solid or open circles: starting points ('heels")

7 or targets (respectively); colors coordinated between R cells and targets. T3': target of fate-altered

8 R3s; T0: target located within the bundle of interest (though targeted by R cells from other bundles

9 in NSP wiring). Bottom panels: confocal images of representative bundles. Photoreceptor growth

10 cones were segmented, pseudo-colored, and intensity scaled for visualization (Methods). Red:

11 sev $>$ RFP expression; green: $m \delta$-GFP expression; white: Fasciclin 2 (FasII) antibody staining.

12 White ellipses: targets. Scale bar: $5 \mu \mathrm{m}$. 
A Identify R-Cell Starting Points

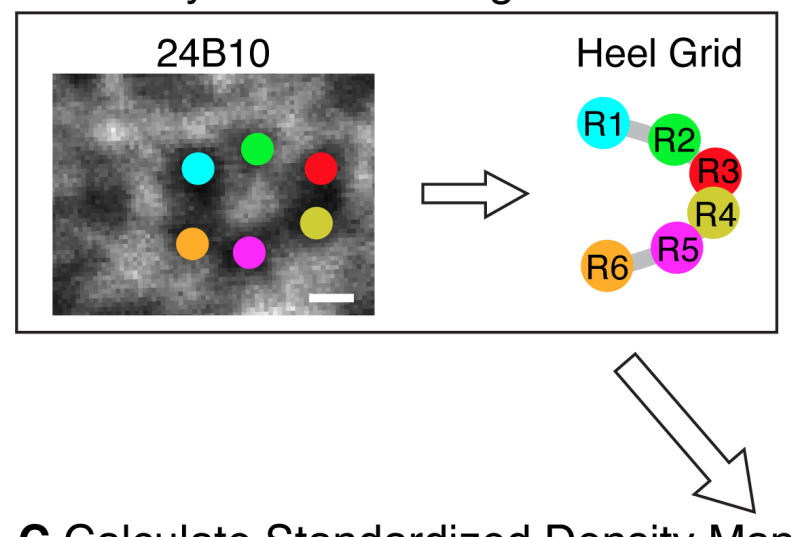

B Identify Positions of Targets

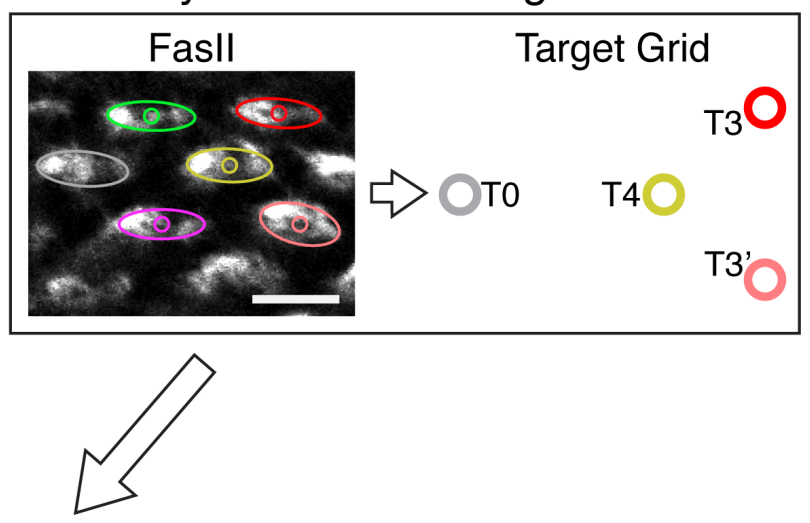

C Calculate Standardized Density Map

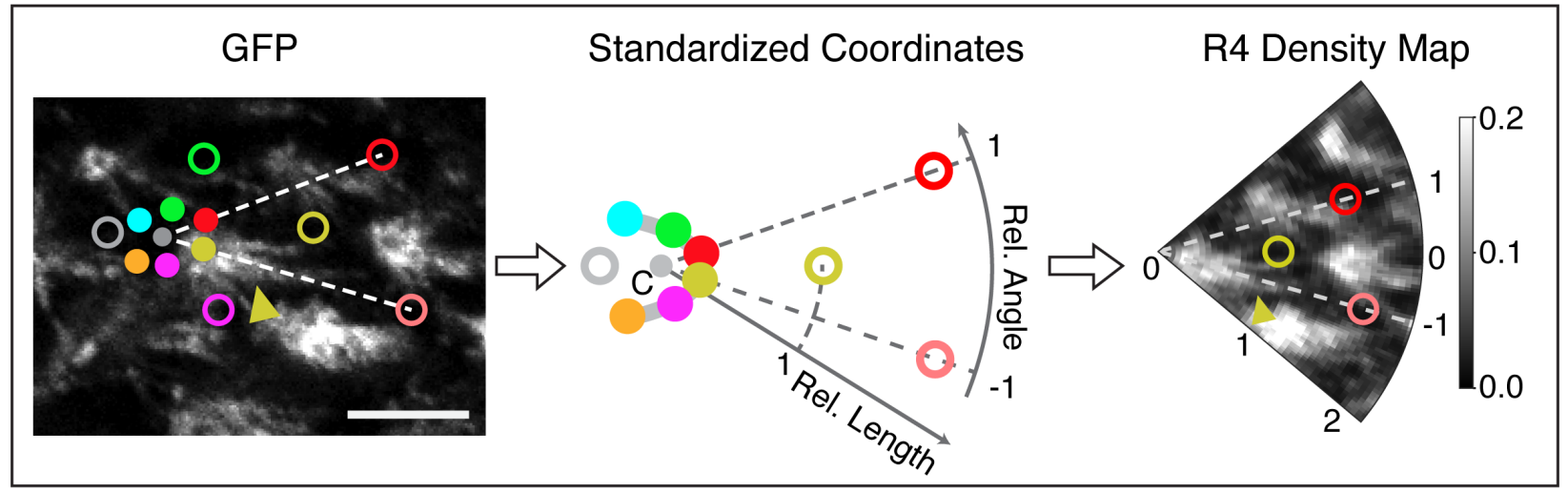

Figure 2. Establishment of standardized coordinates for comparison of growth cone

\section{7 extension.}

18 Overview of the image quantification process. (A) The heel grids (i.e. R-cell starting points) are

19 identified (via 24B10 antibody labeling R-cell membranes; Methods). (B) The target grid is

20 identified (via FasII antibody labeling L-cells membranes; Methods). (C) In each region, (left) the

21 annotation (from A-B) is used (middle) to produce standardized coordinates that are used (right)

22 to transform confocal images into standardized density maps. This transformation allows relative

23 lengths (radial coordinate) and angles (angular coordinate) of extending growth cones to be

24 compared across regions. Images: confocal images of a bundle region were sampled from one 
bioRxiv preprint doi: https://doi.org/10.1101/2020.10.13.337865; this version posted October 13,2020 . The copyright holder for this preprint (which was not certified by peer review) is the author/funder, who has granted bioRxiv a license to display the preprint in perpetuity. It is made available under aCC-BY 4.0 International license.

25 wild-type fly at $26 \mathrm{hrs}$ APF. Images were cropped, re-oriented and intensity scaled for visualization

26 (Methods). Scale bars: $5 \mu \mathrm{m}$ for FasII and GFP images; $1 \mu \mathrm{m}$ for 24B10 image.

27 
A Targets (Fasll) R cells (24b10)
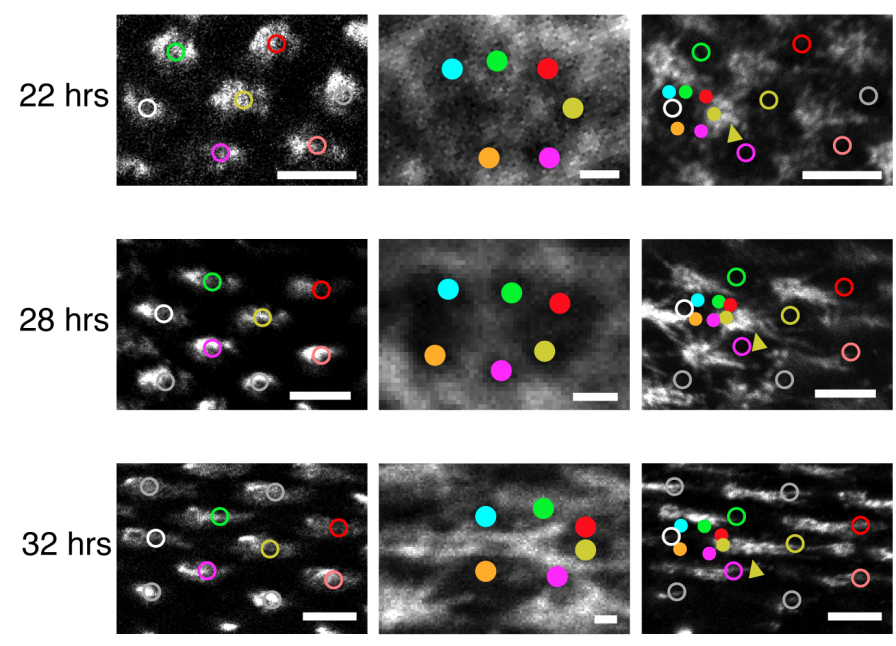

B
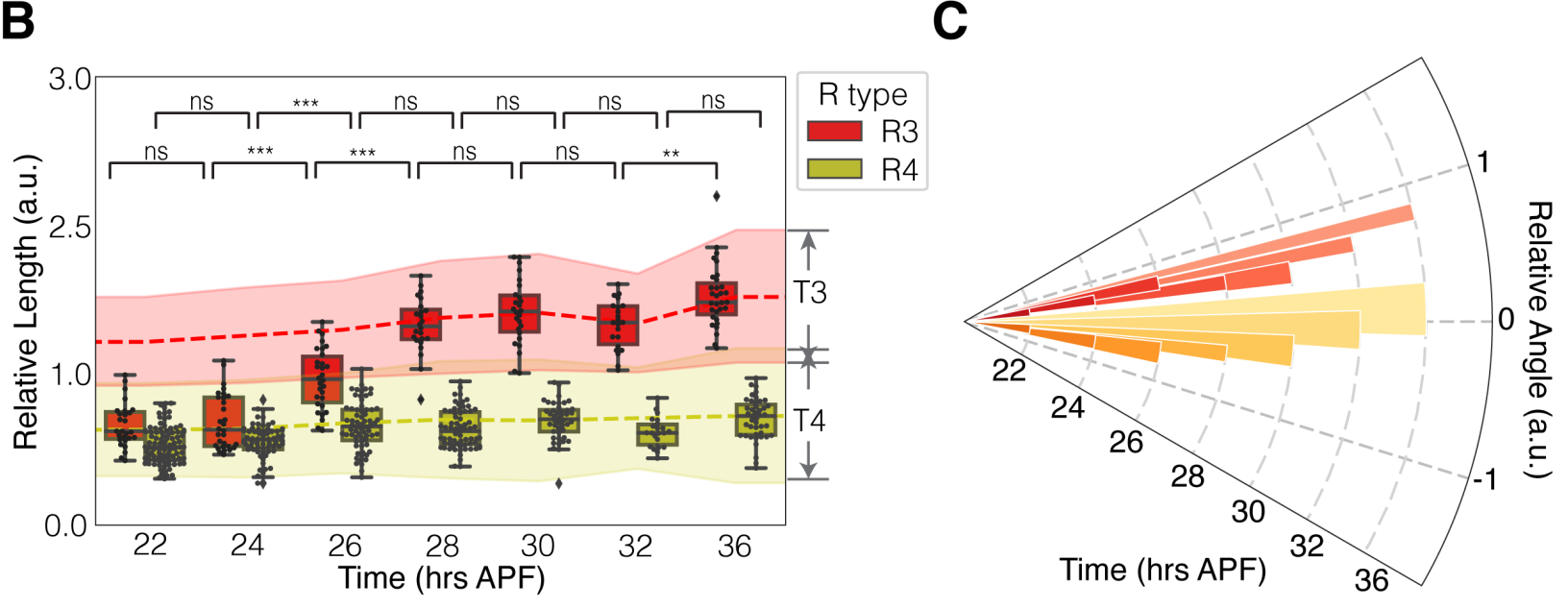

Figure 3. Wild-type R3s and R4s exhibit asymmetric speeds but symmetric directions of

32 (A) Representative images of bundles from wild-type flies at 22, 28, and $32 \mathrm{hrs}$ APF (See Figure

333 - figure supplement 1 for examples at different timepoints). Left: Confocal images of example

34 bundles. From left to right: FasII channel labeling the target cells; 24B10 channel labeling

35 membrane of all R cells; GFP channel labeling membrane of R4 cells; RFP channel labeling

36 membrane of R3 or R4 cells. Right: Density maps of GFP (R4 cells) and RFP channel (R3 or R4 
37 cells) after coordinate transformation. Images were cropped, re-oriented and intensity scaled

38 individually for visualization (Methods). R-cells and targets indicated and colored as in Figure 1;

39 white circles: T0; gray circles: other targets. Yellow arrowheads: R4 growth cones; red arrows: R3

40 growth cones. Scale bars: $5 \mu \mathrm{m}$ for FasII, GFP and RFP images; $1 \mu \mathrm{m}$ for $24 \mathrm{~B} 10$ images. (B)

41 Change in relative lengths of wild-type R3 and R4 growth cones over time. Red and yellow dashed

42 lines: mean of the center of target positions for T3 and T4, respectively. Error bars of the dashed

43 lines: mean of the upper and lower boundaries of targets. Significance: calculated using a two-

44 sided Mann-Whitney test with $\mathrm{p}$ values adjusted by Bonferroni method. ns: $\mathrm{p}>0.5, *: 0.01<\mathrm{p}<$

$450.05, * *: 0.001<\mathrm{p}<0.01, * * *: \mathrm{p}<0.001$. Sample sizes (number of bundles) of each time point:

46 R3 growth cones $(n=27,30,30,24,26,22,31)$; R4 growth cones $(n=96,67,67,67,44,22,43)$.

$47 \mathrm{n} \geq 2$ biological replicates for each time point. Supplementary file 1B for p-values. (C) Change in

48 relative angles of wild-type R3 and R4 growth cones over time. Red and yellow bars represent R3s

49 and R4s, respectively. Plotted bars: radial coordinate indicates time; angle coordinate indicates

50 mean relative angle of R3 or R4 growth cones at the given time point; and width indicates standard

51 deviation of angle values at the given time point. Sample sizes as in (B). See Supplementary file

$521 \mathrm{~B}$ for $\mathrm{p}$-values. 

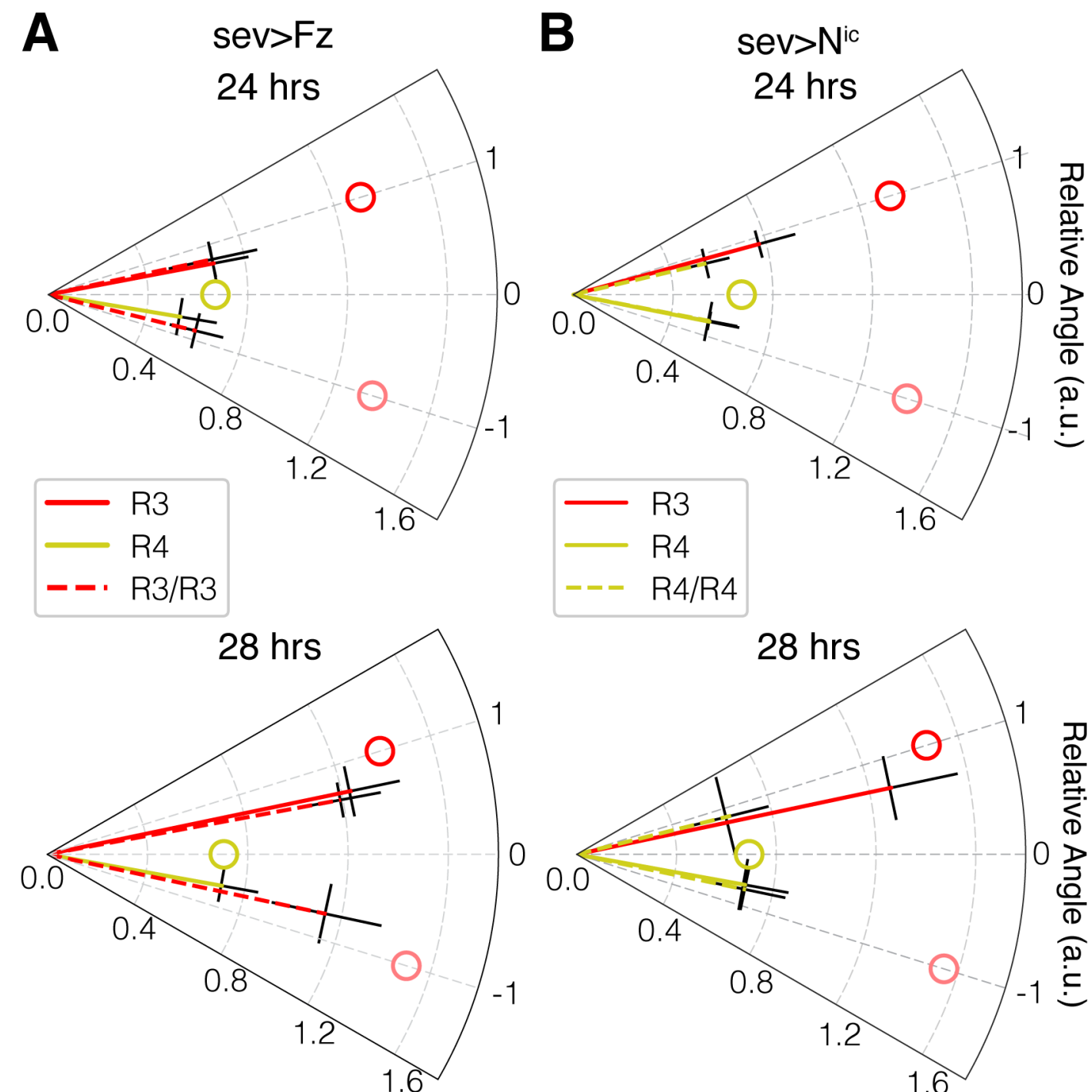

Relative Length (a.u.)

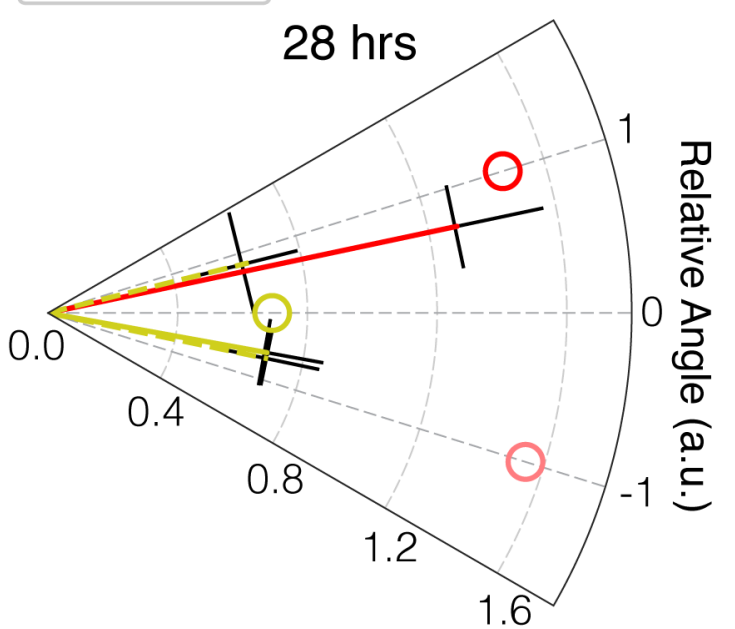

Relative Length (a.u.)

56 Figure 4. Extension speed is responsible for asymmetrical targeting.

57 Polar plots of relative lengths and angles for wild-type-like (R3/R4) and perturbed (R3/R3 or

$58 \mathrm{R} 4 / \mathrm{R} 4)$ bundles under (A) $\operatorname{sev}>F z$ and (B) $\operatorname{sev}>N^{i c}$ conditions at 24 and $28 \mathrm{hrs}$ APF. See Figure 4

59 - figure supplement 1 for representative images of each condition. Radial coordinate: relative

60 length; angle coordinate: relative angle; error bars: standard deviations for length or angle. Solid

61 lines: control bundles; dashed lines: perturbed bundles. Open circles: mean of the center of target

62 positions. Sample numbers for growth cones at wild-type R3 position, wild-type R4 position, 
63 perturbed R3 position, and perturbed R4 position, respectively: $\operatorname{sev}>F z$ at $24 \mathrm{hrs}(\mathrm{n}=24,38,10$,

$6410)$ or $28 \mathrm{hrs}(\mathrm{n}=52,151,23,23) ; \operatorname{sev}>N^{i c}$ at $24 \mathrm{hrs}(\mathrm{n}=23,46,10,10)$ or $28 \mathrm{hrs}(\mathrm{n}=39,113$,

$6529,29) . \mathrm{n} \geq 3$ biological replicates for each genetics and time point. Supplementary file $1 \mathrm{C}$ for $\mathrm{p}$ -

66 values. 


\section{A}

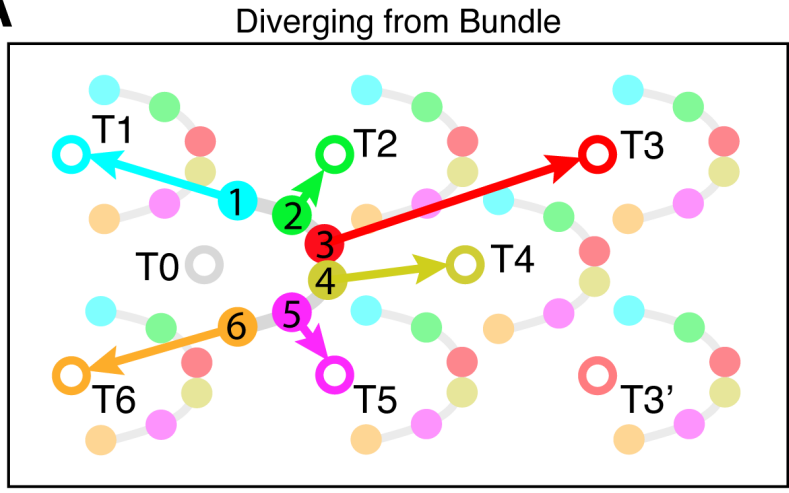

B wiring. APF: “After Puparium Formation”.

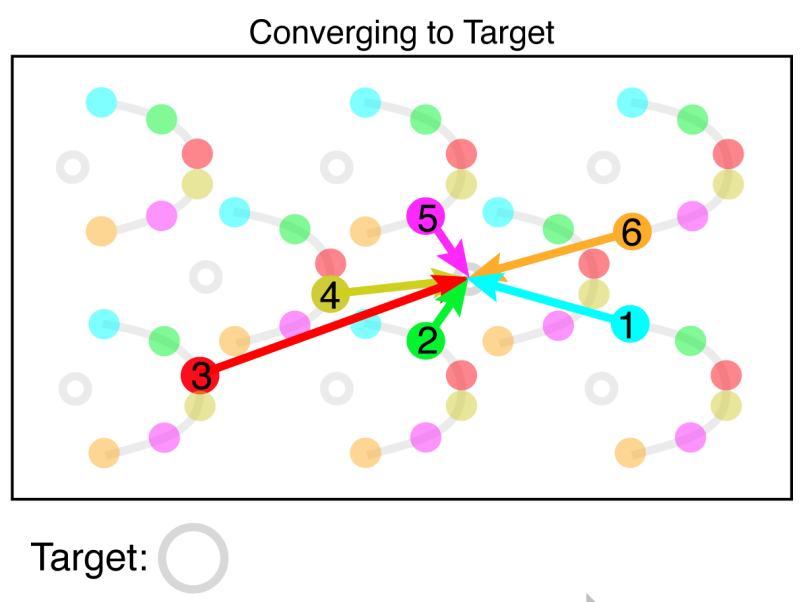

Heel: 9 Target:

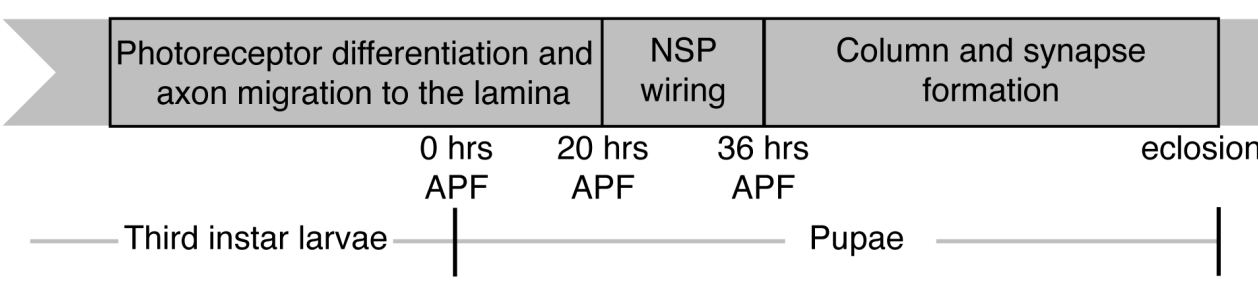

Figure 1 - figure supplement 1. Overview of Neural Superposition.

(A) Schematic of NSP wiring topology. Solid or open circles: starting points ("heels") or targets (respectively). T3': target of fate-altered R3s; T0: target located within the bundle of interest (though targeted by R cells from other bundles in NSP wiring). (B) Schematic for timing of NSP 
A

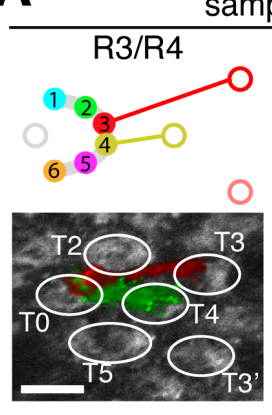

B

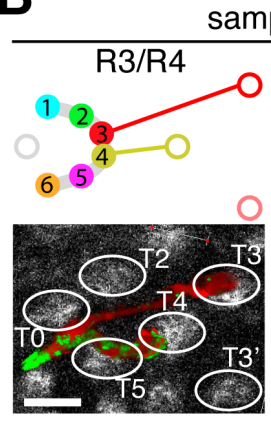

11 Figure 1 - figure supplement 2. Final targeting of $\operatorname{sev}>F z$ and $\operatorname{sev}>N^{i c}$ flies.

12 (A-B) Schematics (top panels) and confocal images (bottom panels) of bundles from three (A)

$13 \operatorname{sev}>F z\left(38 \mathrm{hrs}\right.$ APF) and (B) $\operatorname{sev}>N^{i c}(45 \mathrm{hrs}$ APF) flies. Top panels: schematic of wild-type or

14 altered wiring topology. Solid or open circles: starting points ('heels") or targets (respectively);

15 colors coordinated between R cells and targets. T3': target of fate-altered R3s; T0: target located

16 within the bundle of interest (though targeted by R cells from other bundles in NSP wiring).

17 Bottom panels: confocal images of representative bundles. Photoreceptor growth cones are

18 segmented and pseudo-colored (Methods) and intensity scaled for visualization. Red: $\operatorname{sev}>R F P$

19 expression; green: $m \delta$-GFP expression; white: Fasciclin 2 (FasII) antibody staining. White ellipses:

20 targets. Scale bar: $5 \mu \mathrm{m}$.
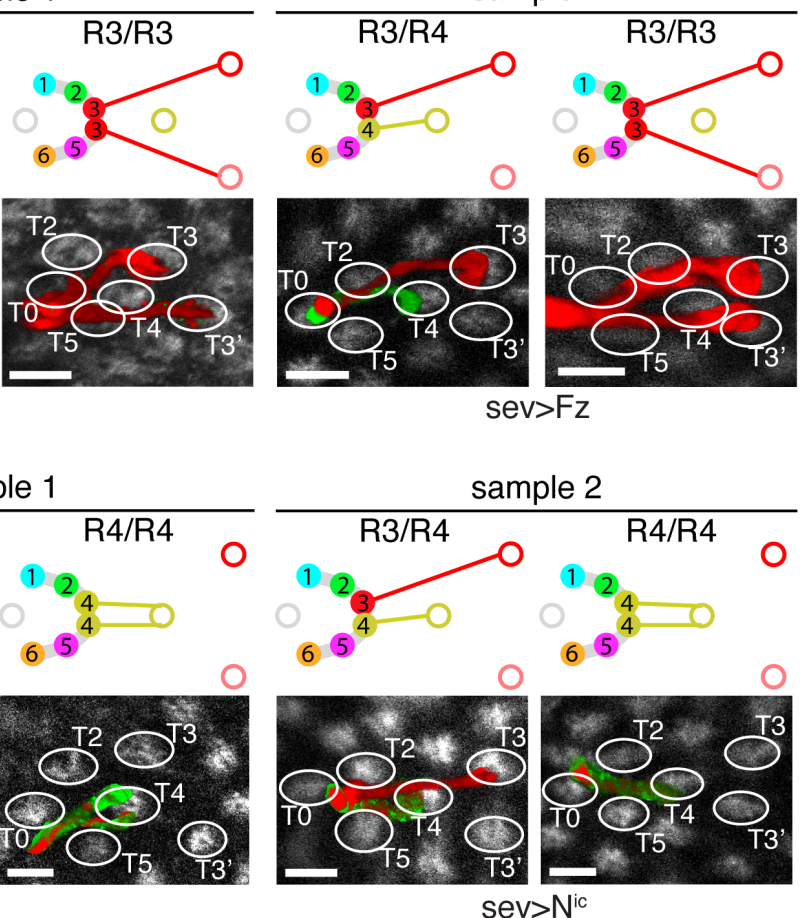
Raw Images
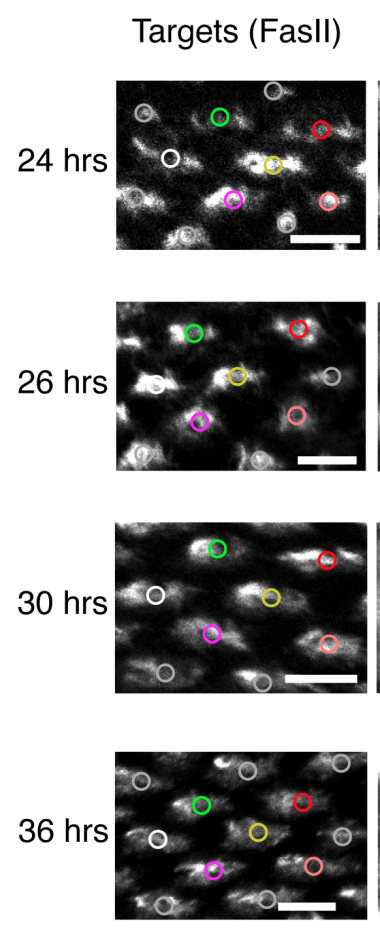

$R$ cells (24B10)
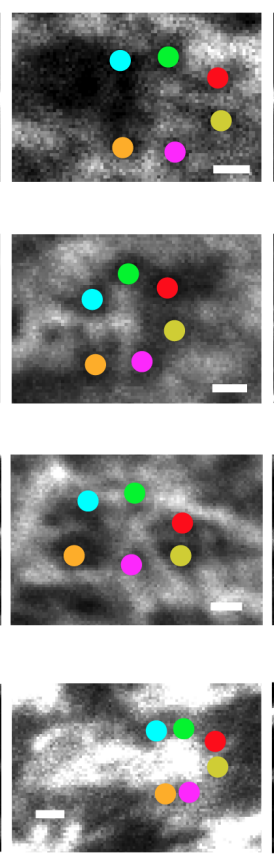

R4 (GFP)
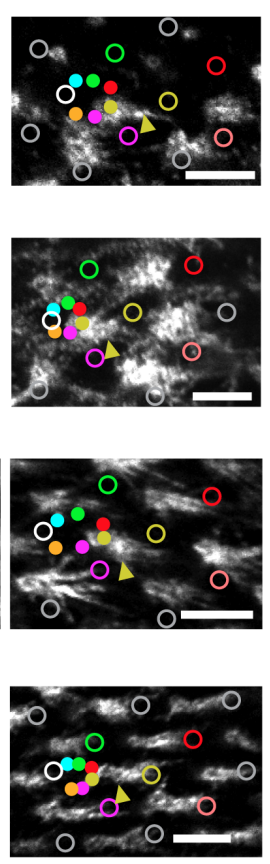
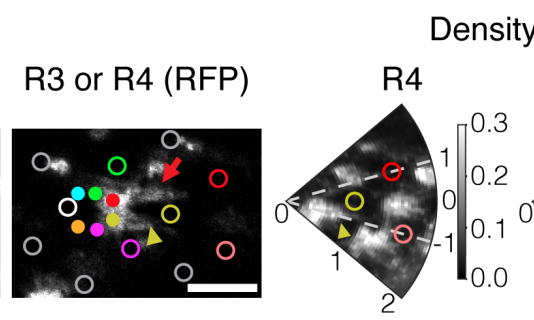

Density Maps
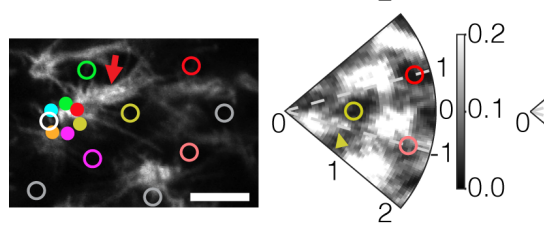

R3 or R4
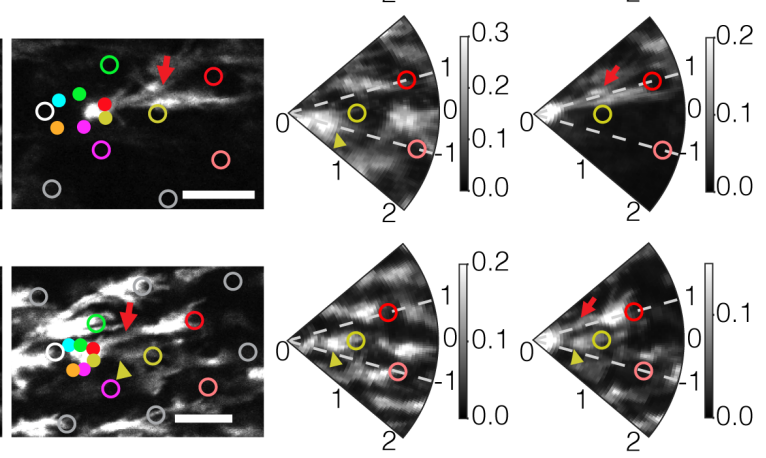

Figure 3 - figure supplement 1. Extension phenotype of wild-type flies over time.

25 Representative images of bundles from wild-type flies at 24, 26, 30, and 36 hrs APF. Left: Raw

26 images of example bundles. From left to right: FasII channel labeling the target cells; 24B10

27 channel labeling membrane of all R cells; GFP channel labeling membrane of R4 cells; RFP

28 channel labeling membrane of R3 or R4 cells. Right: Density maps of GFP (R4 cells) and RFP

29 channel (R3 or R4 cells) after coordinate transformation. For visualization, intensity is scaled

30 differently for each channel and for each sample. R-cells and targets indicated and colored as in

31 Figure 1 - figure supplement 2; white circles: T0; gray circles: other targets. Yellow arrowheads:

32 R4 growth cones; red arrows: R3 growth cones. Scale bars: $5 \mu \mathrm{m}$ for FasII, GFP and RFP images;

$331 \mu \mathrm{m}$ for 24B10 images. 


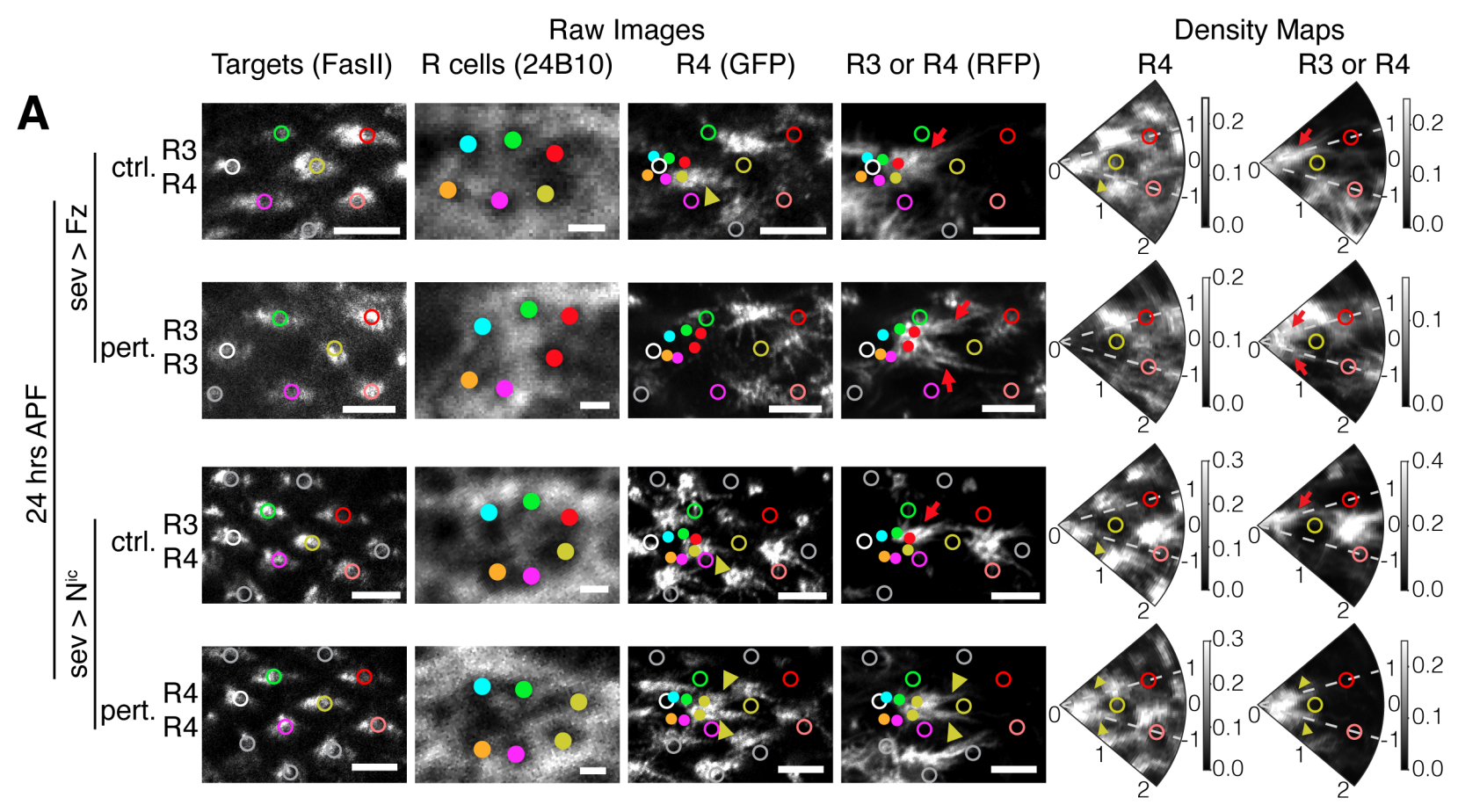

B

37 Figure 4 - figure supplement 1. Extension phenotype of $\operatorname{sev}>F z$ and $\operatorname{sev}>N^{i c}$ flies over time.

38 (A-B) Representative images of wild-type-like (ctrl.) and fate-altered (pert.) bundles in $\operatorname{sev}>F z$

39 and $\operatorname{sev}>N^{i c}$ flies at (A) 24 or (B) $28 \mathrm{hrs}$ APF. Left four panes are confocal images of example

40 bundles. Right two panels are density maps of GFP (R4 cells) and RFP channel (R3 or R4 cells) 
bioRxiv preprint doi: https://doi.org/10.1101/2020.10.13.337865; this version posted October 13, 2020. The copyright holder for this preprint (which was not certified by peer review) is the author/funder, who has granted bioRxiv a license to display the preprint in perpetuity. It is made available under aCC-BY 4.0 International license.

41 after coordinate transformation. Image channels, intensity normalization, annotation and scale bars

42 are as in Figure 3 - figure supplement 1.

43 
A

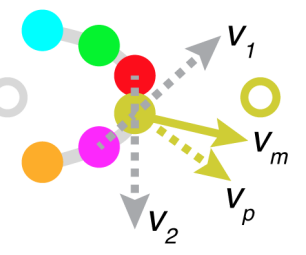

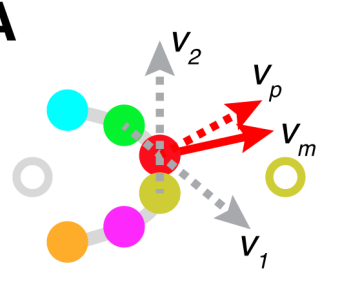

B

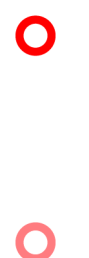

0 $53=0.5$. For the weighted repulsion model, linear regression is performed to get $\alpha$ and $\beta$ that best fit

54 pooled data from wild-type measurements between 22 to $26 \mathrm{hrs}$ APF. R3 regression result: $\alpha=$ 55

Figure 4 - figure supplement 2. Repulsion model for determining growth cone extension angle.

(A) Schematics of repulsion model. For R3, $\overrightarrow{v_{1}}$ and $\overrightarrow{v_{2}}$ represent repulsive forces from R2 and R4, respectively. For R4, $\overrightarrow{v_{1}}$ and $\overrightarrow{v_{2}}$ represent repulsive forces from R5 and R3, respectively. $\overrightarrow{v_{p}}$ : extension direction predicted from simulation; $\overrightarrow{v_{m}}$ : extension direction measured. (B) Difference between predicted and actual extension directions for data from 22, 24 or 26 hrs APF. $\overrightarrow{v_{p}}=\alpha$ $\overrightarrow{v_{1}}+\beta \overrightarrow{v_{2}}$ is used to calculate predicted extension directions. For the equal repulsion model, $\alpha=\beta$ 1.03, $\beta=0.44, \mathrm{R}^{2}=0.75$; $\mathrm{R} 4$ regression result: $\alpha=1.00, \beta=0.64, \mathrm{R}^{2}=0.87$. 